\title{
Integrated Literature Review of Depression in Elderly People
}

Ya Lie Ku ${ }^{1 *}$ and Li-Yu Chung ${ }^{2}$

${ }^{1} R N$, MSN, Associate Professor, College of Nursing, Fooyin University

${ }^{2} R N$, MSN, Instructor, College of Nursing, Fooyin University

\begin{abstract}
The authors conducted a literature review of the depression findings of systematic reviews and meta-analyses in elderly people. The defining characteristics of depression in elderly people include $2-5$ symptoms, namely sleep disorders, changed weight, psychomotor retardation, fatigue, and feelings of worthlessness or guilt within 2 weeks; the critical symptom is either low mood or loss of interest in usual activities. In addition, elderly people with an abnormal status for the items of the Geriatric Depression Scale (GDS) may have experienced recurrent falls twice during the past 2 years. The related factors of depression in elderly people include demographic, physical, psychological, mental, and social factors. Colasanti et al. in 2010 identified four major self-rating depression scales for elderly people: Beck Depression Inventory (BDI), Self-rating Depression Scale (SDS), Center for Epidemiological Studies Depression (CES-D), and GDS. CES-D is a valid scale that can be used to distinguish major depression from emotional disorders among elderly people, and it can also be used in pharmacological trials. The interventions for depression in elderly people include pharmacological and nonpharmacological methods. Regarding pharmacological treatment, the evidence-based literature has demonstrated that duloxetine taken for 8 weeks can alleviate recurrent major depression in elderly people; at the same time, healthcare professionals should pay attention to some side effects. Moreover, herbal medicine has been found to only alleviate post-stroke depression in elderly people. Nonpharmacological interventions are exercise therapies such as yoga; alternative therapies such as touching, intimate massage, music, art, and nature; and cognitive therapy, reminiscence therapy, and psychotherapy.
\end{abstract}

Keywords: Depression; Elderly

\section{Introduction}

According to the World Health Organization (WHO) report by 2020 , depression will be the second leading cause of disability worldwide; currently, depression is very prevalent in developing countries; globally, the second leading cause of disability is depression. Studies have shown that currently, $30 \%$ of elderly people aged more than 65 years have emotional disorders, and $64 \%$ of hospitalized elderly people have depression symptoms such as low mood, sleep disturbance, poor appetite, or pain combined with anxiety [1-5]. Additionally, $36.9 \%$ of 111 elderly patients experienced depression, and the prevalence was $45.7 \%, 36.2 \%$, and $22.2 \%$ in those living in nursing homes, intermediate care facilities, and domiciliary care facilities, respectively [6]. Specifically, $21.7 \%$ of 152 veterans developed depression [7]. Moreover, $34.9 \%$ of community-dwelling elderly veterans in the Yunlin-Chiayi area exhibited depression symptoms; this prevalence rate was higher than that for other communities in Taiwan [8].

Depression exerts severe effects in elderly people, including physical and psychological symptoms and social functioning disorders. Based on research findings accumulated over many years, elderly people with mild, early-diagnosed, and relapsed depression exhibit a high risk of dementia [5]. Furthermore, systematically reviewed the literature on the association of depression symptoms with recurrent falls among the elderly population and demonstrated that elderly people with an abnormal status for four items of the Geriatric Depression Scale (GDS) had experienced recurrent falls twice during the past 2 years [9]. Overall, the depression symptoms and disorders influence their health and are the major factors causing a high risk of suicide; thus, depression represents a heavy burden on the family and society $[3,4,8]$.

\section{Purpose of the Study}

The purpose of this study was to conduct a literature review of the depression findings of systematic reviews and meta-analyses in elderly people.

\section{Methods}

The authors used key word "depression", "elders", and systematic reviews" or meta-analysis" in the CINAHL database from 2010 to 2016.
The 22 articles were identified with the extra 4 articles being indicating as the important papers; therefore, the final 26 papers were integrated to be reviewed.

\section{Findings}

The findings included the defining characteristics, related factors, assessment scales, and interventions for depression in elderly people.

\section{Defining characteristics of depression in elderly people}

According to the diagnostic and statistical manual of mental disorders, fourth edition, people experiencing 2 to 5 symptoms, including low mood and loss of interest and pleasure in usual activities, within 2 weeks are diagnosed with mild depression [10]. The diagnostic criteria for depression are the same for adults and elderly people; that is, for 2 weeks, at least five symptoms occur, including low mood, loss of interest in usual activities, lethargy or insomnia, gained or lost weight, psychomotor retardation, fatigue, feelings of worthlessness or guilt, suicidal ideation, or decreased concentration, but one critical symptom must occur: either low mood or loss of interest in usual activities [5]. Additionally, the diagnostic criteria for depression as low mood, loss of interest and pleasure, insomnia, fatigue, discontinued activities, and feelings of worthlessness and despair [4]. Furthermore, elderly people with an abnormal status for four items of GDS had experienced recurrent falls twice during the past 2 years, and these people were found to exhibit depression [9]. Finally, sleep disturbance as the most frequent symptom of depression in elderly people [11].

*Corresponding author: Ya Lie Ku, Department of Nursing, No.151, Chin-Hsueh Rd., Ta-Liao District, Kaohsiung City 83102, Taiwan, ROC, Tel: (O) (07)78111515312 (H) (07)7832096 Cellphone: 0931381365, E-mail: ns126@fy.edu.tw

Received August 22, 2017; Accepted September 04, 2017; Published September 11, 2017

Citation: Ku YL (2017) Integrated Literature Review of Depression in Elderly People. J Gerontol Geriatr Res 6: 446. doi:10.4172/2167-7182.1000446

Copyright: () $2017 \mathrm{Ku} \mathrm{YL}$. This is an open-access article distributed under the terms of the Creative Commons Attribution License, which permits unrestricted use, distribution, and reproduction in any medium, provided the original author and source are credited. 


\section{Related factors of depression in elderly people}

The related factors of depression in elderly people include demographic, physical, psychological, mental, and social factors. Demographic factors such as age, gender, marital status, religion and faith, educational level, occupation, economic status, living patterns, hospitalization duration, and changed environment are all related to depression in elderly people $[1,7,8,11]$. Physical factors such as acute myocardial infarction, heart failure, stroke, cerebrovascular accident, hypothyroidism, crippling and/or disabling diseases, dementia and neurodegenerative diseases, bone fractures, osteoporosis, arthritis, and chronic illness; dysfunction in physical attributes, such as blood sugar and cardiovascular system imbalance, hydroelectrolytic disturbances, or lower liver and kidney functions; and lower daily activity score are related to depression in elderly people $[1-3,6,7,10]$.

Specifically, among the elderly population, early depression is caused by family history, and late depression is caused by degenerative lesions in the white matter in the brain or brain vascular infarction [5]. Additionally, bad habits such as smoking and drinking and a selfperceived poor health status are also related to depression in elderly people $[7,8]$. Psychological, mental, and social factors related to depression in elderly people include a history of general anxiety, specific phobia, compulsive behaviors, major depression, feelings of loneliness and lack of intimacy in relationships because of living in long-term care facilities without sufficient support, mental senescence, lost role or role changes because of deaths of family members or retirement, and social difficulties $[2,3,10,11]$.

\section{Assessment scales of depression in elderly people}

Systematically reviewed assessment scales for depression in elderly people and identified four major self-rating depression scales for elderly people: Beck Depression Inventory (BDI) [2,12], Self-rating Depression Scale (SDS) [2,13], Center for Epidemiological Studies Depression (CES-D) $[2,14]$, and GDS [2,15]. BDI consists of 21 items and evaluates the behavioral symptoms of depression. However, it is not sensitive to clinical variation and is thus not used in pharmacological trials. SDS consists of 20 items and is the main scale used to assess general depression in adults; it is used for false positive screening for seniors aged more than 70 years [2]. Furthermore, CED-D consists of 20 questions and distinguishes between clinical depression and emotional disorders in elderly people; GDS consists of 30 items and is a valid screening scale for major and minor depression; however, the scale for minor depression is not used in pharmacological trials [2].

\section{Interventions for depression in elderly people}

The interventions for depression in elderly people include pharmacological and nonpharmacological methods. From the pharmacological perspective, three stages of medical treatment for depression in the elderly population: the acute stage, the relapse stage, and the prevention stage [16]. The purpose of the first stage is to alleviate the symptoms, the second stage aims to maintain healthy function, and the third stage prevents the recurrence of depression [16]. However, at the same time, healthcare professionals should assess the chronic illnesses of patients using depression medications and their past depression symptoms and medications in terms of categories, doses, and side effects [16]. Specifically, duloxetine taken for 8 weeks can alleviate recurrent major depression in people aged 65 years or older with major depressive disorder, but it also increases the risk of side effects such as thirst, constipation, diarrhea, and dizziness [17].

Additionally, systematically reviewed 298 potentially relevant studies and selected 13 randomized controlled trials (RCTs) with fitting the inclusion criteria to analyze the efficacy and safety of Gan Mai Da Zao (GMDZ) decoction for depression [4]. Results identified that compared with anti-depression medicines, herbal medicine (Gan Mai Da Zao decoction) only alleviates post-stroke depression in elderly people; however, it could not provide evidence for major depression, post-surgical depression, or depression in the elderly in terms of the superiority of GMDZ decoction over anti-depressant therapies [4].

From the nonpharmacological perspective, systematically reviewed 461 clinical trials and reported that exercise therapy decreases the depression symptoms of elderly people and improves their self-esteem and quality of life [18]. Similarly, regular exercise could prevent the depression symptoms of 585 community-dwelling elderly people in Taiwan, regardless of gender, health status, and chronic illness [1]. Specifically, a systematic review showed that yoga could significantly reduce the depressive symptoms of elderly participants and improve their quality of sleep after 6 months both for elders living in institutions and the community [11]. Moreover, yoga exercises significantly decreased depression, sleep disturbance, and daytime dysfunction in 38 elderly people after 6 months, and these outcomes were more favorable than those of the control group of 31 elderly people [19].

\section{Discussion}

Alternative therapies are also effective for treating depression symptoms in elderly people. Regarding physical contact therapy, the depression and agitated behaviors of 12 older people with dementia significantly improved after they received robot- assisted therapy involving PARO for 40 minutes, twice a week for 4 weeks; simultaneously, their verbal and body interactions were facilitated by nurses [20]. Similarly, 15-day intimate massage including head, neck, shoulders, hands, palms, fingers, and back could reduce depression and anxiety in 6 elderly people living in institutions [21].

Regarding music therapy, hospitalized elderly patients who listened to music for 30 minutes once a day during days 3 to 7 at a medical center had significantly reduced depression as well as smooth the heart rate and blood in the experimental group $(\mathrm{N}=36)$, comparing with the control groups $(\mathrm{N}=33)$ [3]. Regarding art therapy, 29 elderly patients who participated in 1-hour sessions of 12 artistic activities for 6 weeks, twice a week, showed significantly reduced depression and improved self-esteem [22]. Regarding nature therapy, the evaluating 10 elderly patients living in a nursing home who underwent a 10 -week program of indoor horticultural therapy, with one 1.5-hour session per week; they reported that depression and loneliness in these patients significantly improved, along with improvements in the four positive themes of social connection, anticipation and hope, sense of achievement, and companionship [23].

Furthermore, regarding cognitive therapy, the assessing 23 elderly people with dementia who underwent individualized learning therapy for 30 minutes, twice a week; they showed that this therapy significantly improved cognitive functions and decreased neuropsychiatric symptoms such as hallucinations, depression, apathetic expression, irritability, bizarre behavior, and sleep disorders by the seventh week [24]. Similarly, in a meta-analysis, the reminiscence therapy significantly improved depression, self-esteem, and life satisfaction in 852 elderly people [25]. Finally, regarding psychotherapy, a meta-analysis of 17 trials; they reported a medium effect size of psychotherapy for decreasing depression symptoms in elderly people, and the effect was maintained at follow-up [26]. 


\section{Conclusion and Suggestions}

According to the integrated review, the defining characteristics of depression in elderly people include 2 to 5 symptoms, namely sleep disorders, changed weight, psychomotor retardation, fatigue, and feelings of worthlessness or guilt within 2 weeks. The critical symptom is either low mood or loss of interest in usual activities. Moreover, elderly people with an abnormal status for the items of GDS may experience recurrent falls twice during the past 2 years. The related factors of depression in elderly people include demographic, physical, psychological, mental, and social factors. Four major selfrating depression scales for elderly people, including BDI, SDS, CES-D, GDS, and CES-D is a valid scale that can be used to distinguish major depression from emotional disorders among elderly people, and it can also be used in pharmacological trials. The interventions for depression in elderly people include pharmacological and nonpharmacological methods.

Regarding pharmacological treatment, the evidence-based literature has demonstrated that duloxetine taken for 8 weeks can alleviate recurrent major depression in elderly people; at the same time, healthcare professionals should pay attention to some side effects. Moreover, herbal medicine (Gan Mai Da Zao decoction) only alleviates post-stroke depression in elderly people. Nonpharmacological interventions are exercise therapies such as yoga; alternative therapies such as touching, intimate massage, music, art, nature, as well as cognitive, reminiscence, and psychotherapy. Based on the literature review findings, the authors recommend that future studies should develop a comprehensive depression assessment scale for elderly patients that include the related factors and defining characteristics of depression in elderly people. Based on the evidence-based literature, more nonpharmacological interventions should be administered to elderly patients with depression to improve their quality of life.

\section{References}

1. Chang SH, Chien NH, Chen MC (2016) Regular exercise and depressive symptoms in community-dwelling elders in northern Taiwan. J Nurs Res 24: 329-336.

2. Colasanti V, Marianetti M, Micacchi F, Amabile GA, Mina C (2010) Tests for the evaluation of depression in the elderly: A systematic review. Arch Gerontol Geriatr 50: 227-230.

3. Hou HM, Yang SH (2014) The effects of listening to music on depression and physiological parameters of hospitalized elderly patient. VGH Nursing 31: 295-305.

4. Jun JH, Choia TY, Leea JA, Yuna KJ, Leea MS (2014) Herbal medicine (Gan Mai Da Zao decoction) for depression: A systematic review and meta-analysis of randomized controlled trials. Maturitas 79: 370-380.

5. Weng CF, Lin KP, Chan DC (2014) Geriatric depression and cognitive impairment. J Nurs Heal Res 25: 158-164.

6. Huang HT, Chuang YH, Hsueh YH, Lin PC, Lee BO, et al. (2014) Depression in older residents with stroke living in long-term care facilities. J Nurs Res 22: 111-118.

7. Chang TY, Chueh KH (2011) Relationship between elderly depression and health status in male veterans. J Nurs Res 19: 298-304.
8. Kung HW, Lu HY, Huang YC, Lo YF, Yan YH, et al. (2014) Relationship between depressive symptoms and health status for community- dwelling elderly veterans in the Yunlin-Chiayi area. Chang Gung Nurs 25: 243-256.

9. Launay C, De DeCker L, Annweiler L, Kabeshova A, Fantino B, et al. (2013) Association of depressive symptoms with recurrent falls: A cross-section elderly population based study and a systematic review. J Nutr Health Aging 17: 152-157.

10. Polyakova M, Sonnabend N, Sander C, Mergl R, Schroeter ML, et al. (2014) Prevalence of minor depression in elderly persons with and without mild cognitive impairment: A systematic review. J Affect Disord 152-154: 28-38.

11. Wang YY, Chang HY, Lin CY (2014) Systematic review of yoga for depression and quality of sleep in the elderly. Nurs 61: 85-92.

12. Beck AT, Ward CH, Mendelson M, Erbaugh J (1961) An inventory for measuring depression. Arch Gen Psychiatry 4: 561-571.

13. Zung WWK (1965) A self-rating depression scale. Arch Gen Psychiatry 12: 63-70.

14. Radloff LS (1977) The CES-D scale: A self-report depression scale for research in the general population. Appl Psychol Meas, 1: 385-401.

15. Burke WJ, Roccaforte WJ, Wengel SP (1991) The short form of the geriatric depression scale: A comparison whit the 30 -item form. J Geriatr Psychiatry Neurol 4: 173-178.

16. Frank C (2014) Pharmacologic treatment of depression in the elderly. Can Fam Physician 60: 121-126.

17. Tham A, Jonsson U, Andersson G, Söderlund A, Allard P, et al. (2015) Efficacy and tolerability of antidepressants in people aged 65 years or older with major depressive disorder- A systematic review and a meta-analysis. J Affected Disorder 205: 1-12.

18. Park SH, Han KS, Kang CB (2014) Effects of exercise programs on depressive symptoms, quality of life, and self-esteem in older people: A systematic review of randomized controlled trials. Appl Nurs Res 27: 219-226.

19. Chen KM, Chen MH, Lin MH, Fan JT, Lin HS, et al. (2010) Effects of yoga on sleep quality and depression in elders in assisted living facilities. J Nurs Res 18: 53-61.

20. Hu HF, Wang CH, Chang SM, Huang HC, Lai ZY, et al. (2014) Preliminary study on the effects of robot-assisted therapy on depression and agitated behaviors among older people with dementia. VGH Nurs 31: 379-387.

21. Hsu CH, Yang ML (2010) The effect of intimacy massage on reducing depression and anxiety in the institutionalized elders: A single subject design. $\mathrm{J}$ Nurs Healthcare Res 6: 54-64.

22. Wen HN, Wu HL, Kuo CL, Liu WM (2015) The effects of using artistic activities on improving depression and self-esteem among older people in long-term care institutes. J Nurs Healthcare Res 11: 267-276.

23. Chen YM, Ji JY (2015) Effects of horticultural therapy on psychosocial health in older nursing home residents: A preliminary study. J Nurs Res 23: 167-171.

24. Chen MM, Tsai Lj, Chao SY, Clark MJ (2016) Study on the effects of individualized learning therapy on cognitive function and behavioral and psychological symptoms of dementia in the institutionalized older adults. J Nurs Res 24: 300-310.

25. Zhang SJ, Hwu YJ, Wu PI, Chang CW (2015) The effects of reminiscence therapy on depression, self-esteem and life satisfaction on institutionalized older adults: A meta-analysis. J Nurs Healthcare Res 11: 33-42.

26. Cody RA, Drysdale K (2013) The effects of psychotherapy on reducing depression in residential aged care: A meta-analytic review. Clin Gerontologis 36: 46-69. 